\title{
Optical and near-infrared flares in GRB afterglows
}

\author{
Thomas Krühler \\ Dark Cosmology Centre, Niels Bohr Institute, University of Copenhagen, Juliane Maries Vej \\ 30, 2100 Copenhagen, Denmark. \\ email: tom@dark-cosmology.dk
}

\begin{abstract}
Among the diversities in the very early evolution of GRB afterglows are bright optical/near-infrared flares before or superimposed onto an otherwise smoothly decaying afterglow light curve. A lot has been learned about GRBs by using an optical flare or lack thereof as a diagnostic of the emission mechanisms and outflow conditions. In this contribution I will review the observational properties of rising and decaying light-curves in GRB afterglows, discuss their possible physical origins, and highlight in which way they help in understanding GRB and afterglows physics.
\end{abstract}

Keywords. gamma rays: bursts

\section{Introduction}

The launch of the Swift satellite (Gehrels et al. 2004) in 2004 opened a new field of gamma-ray burst (GRB) afterglow physics. With its precise localization by the Burst Alert Telescope, rapid slewing capabilities and early follow-up with two instruments in the X-ray and ultraviolet/optical regime, studies of the early afterglow phase were possible for the first time with larger sample statistics of around 100 per year.

The optical/near-infrared (NIR) afterglow shows significant diversity in its early evolution. While the late phases $(t \gtrsim 1$ day) of the afterglow of long GRBs are generally well described with smoothly decaying power-laws with temporal indices between $t^{-0.5}$ to $t^{-2.5}$, and additional components from associated supernovae or host galaxies, the early afterglow ( $t \lesssim 1$ day) often shows epochs of flaring, or more generally, rise and decay (see Fig. 1).

\section{Reverse shocks and lack thereof}

After the prompt internal shock phase, the optical afterglow light curve is composed of two different emission components. The reverse shock (RS) propagating into the ejecta and the forward shock (FS) travelling into the surrounding medium (e.g., Zhang et al. 2003). Rapid optical observations of the early transition phase between prompt and afterglow emission can constrain the nature of the outflow (e.g., Nakar \& Piran 2004). Baryonic ejecta are expected to produce an optical flash, that can be associated with a RS. The characteristic observational signature of a reverse shock is a relatively steep decline $\left(\propto t^{-2}\right)$, that is too fast for the standard afterglow emission. A Poynting flux dominated afterglow, however, should preferentially show the FS emission.

The hydrodynamical calculations from the fireball model have succeeded in describing the generic afterglow of GRBs from several minutes to days post burst. However, the majority of bursts does not show bright optical flashes and apparently lack a strong RS 


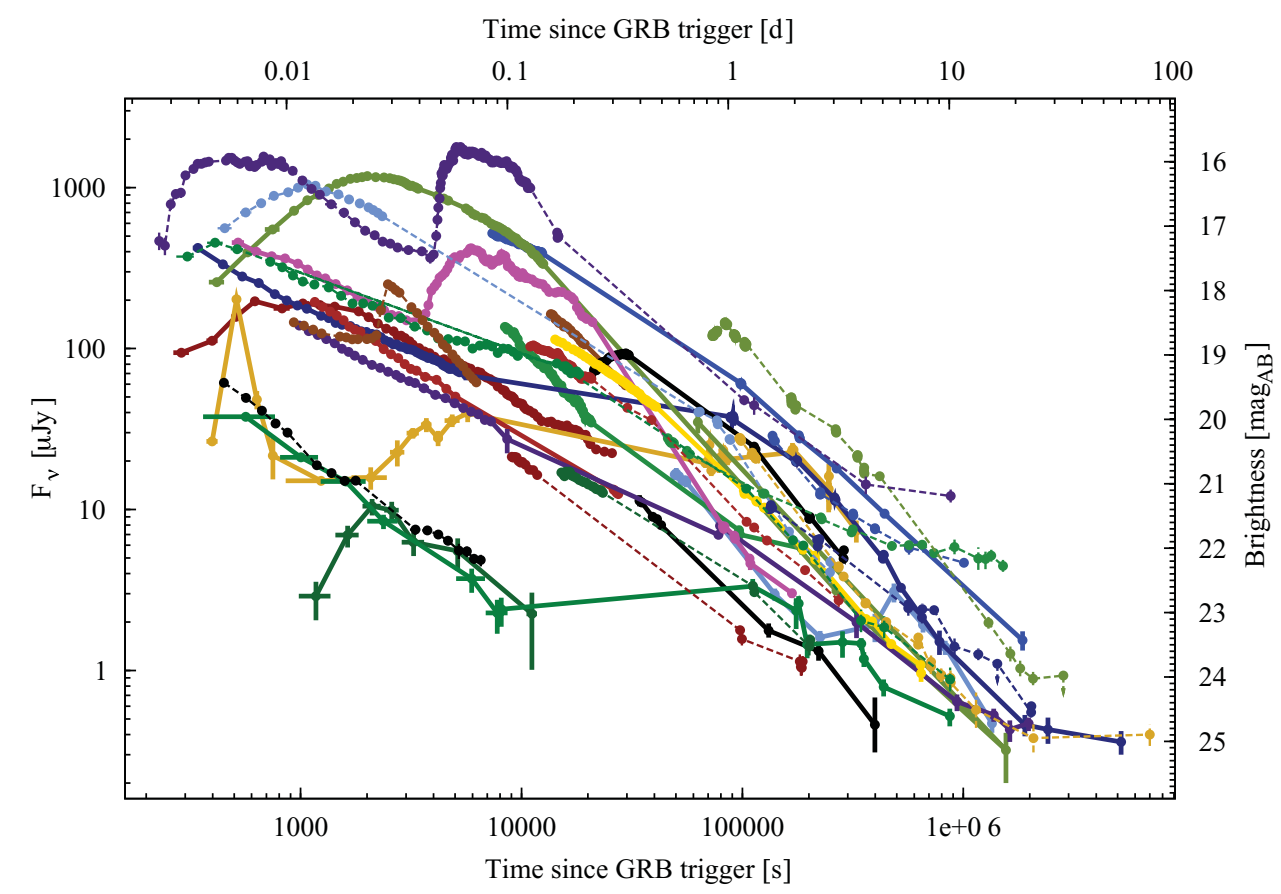

Figure 1. Selection of observed optical light-curves for a number of GRB afterglows obtained with the Gamma-ray Optical Near-infrared Detector (GROND, Greiner et al. 2008). Note the considerable variety in the early evolution of optical/NIR afterglows.

emission component (Roming et al. 2006, Kann et al. 2010). This fact provides observational support that the strength of the optical emission from the RS may be weaker than previously calculated, or that the RS is not radiated in the optical wavelength regime. The properties of the magnetic fields in the ejecta arguably play a crucial role, and might shift the typical synchrotron emission of the reverse shock out of the optical wavelength bands (e.g., Beloborodov 2005).

\section{The onset of the afterglow}

The optical/NIR light curve is in many cases dominated by an early increase in brightness (e.g., Molinari et al. 2007). This early rise is achromatic (see Fig. 2). Thus a movement of the characteristic synchrotron frequency through the observed optical bands, as well as dust destruction are readily ruled out as the origin of the initial rise (see e.g., Krühler et al. 2009b, Perley et al. 2010). The observational characteristics of this afterglow onset are a temporal rise of approximately $\propto t^{0.5-3}$ and a very smooth turnover to the subsequent decay (see Fig. 2).

The early rise in the optical afterglow light-curve is generally attributed to the onset of the forward shock emission. This happens when the swept up medium efficiently decelerates the ejecta. From the time of the light curve peak (typically at few tens to hundreds of seconds), physical parameters of the outflow, such as the initial bulk Lorentz factor $\Gamma_{0}$ or the deceleration radius can be constrained. The early optical afterglow hence provides a robust measurement and confirmation of the ultra-relativistic nature of the GRB phenomenon, yielding values of $100 \lesssim \Gamma_{0} \lesssim 500$, and deceleration radii of $10^{16}$ to $10^{17} \mathrm{~cm}$ with only a weak dependence on the uncertain micro-physical parameters. 


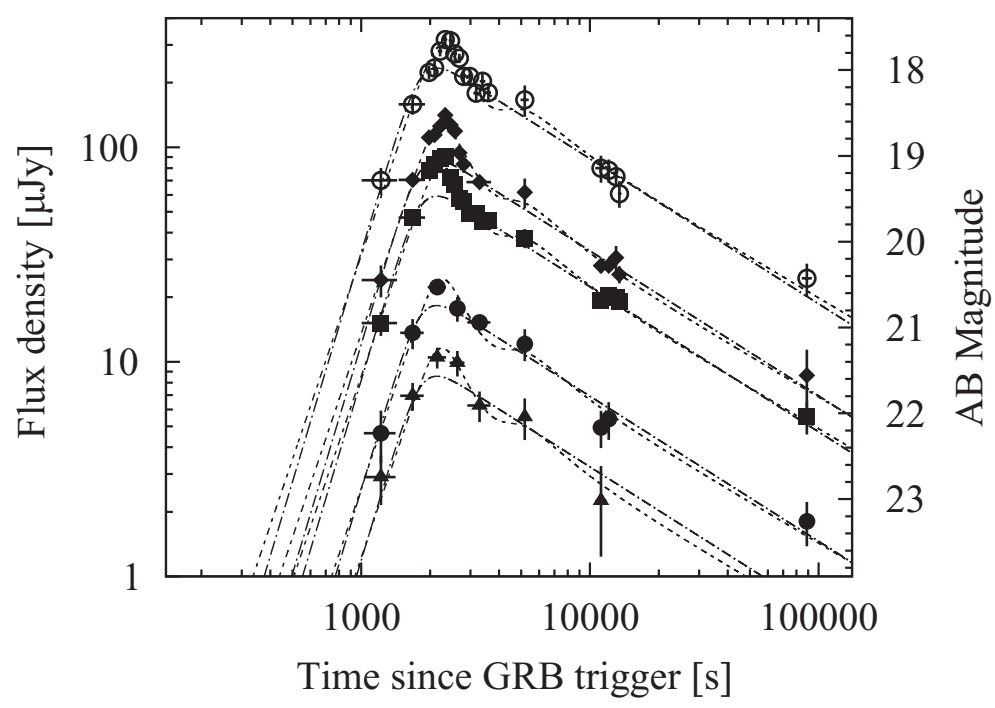

Figure 2. Optical/NIR light-curve of the afterglow of GRB 070802. Five different filters from the GROND instrument $(r z J H K)$ are shown, illustrating the achromatic nature of the rise and decay. Adapted from Krühler et al. (2008).

Even higher Lorentz-factors approaching or possibly exceeding $\Gamma_{0} \sim 1000$ arguably exist. Optical follow-up observations are in those cases however not rapid enough to catch the peak of the afterglow emission at a few seconds after the initial $\gamma$-ray trigger.

Alternatively, an initial achromatic rise of the afterglow might be caused by an off-axis location of the observer with respect to the outflow geometry. Because of the relativistic beaming of the decelerating ejecta, an observer located off-axis to the central jet will see a rising optical afterglow light curve at early times. The steepness of the rise would then be characteristic of the off-axis angle and the jet structure: the farther the observer is located from the central emitting cone or the faster the energy per solid angle decreases outside the jet, the shallower is the observed rise in a structured jet model (Panaitescu \& Vestrand 2008). This scenario is particularly appealing for soft or sub-energetic events such as X-ray flashes, or X-ray rich bursts. A unified picture that attributes both, prompt and early afterglow emission to the observer's viewing angle could be obtained in this scenario.

\section{True flares - fast rise and fast decays}

In some cases, early optical observations reveal a light-curve morphology that is remarkably similar to the X-ray flares, which are detected in approximately $50 \%$ of all X-ray afterglows (e.g., Burrows et al. 2005). These morphologies are characterized by a very fast rise and similarly fast decay (see Fig. 3). Furthermore, these optical flares show strong spectral evolution and time lags, and are in many cases correlated with contemporaneous X-ray flares. There can also be multiple optical/NIR flares for a single event (Krühler et al. 2009a).

This strongly suggests late central engine activity as the common origin. The optical/NIR flares with fast rise and fast decay would thus be the soft tail of emission correlated with late internal shocks. This connection provides additional evidence that 


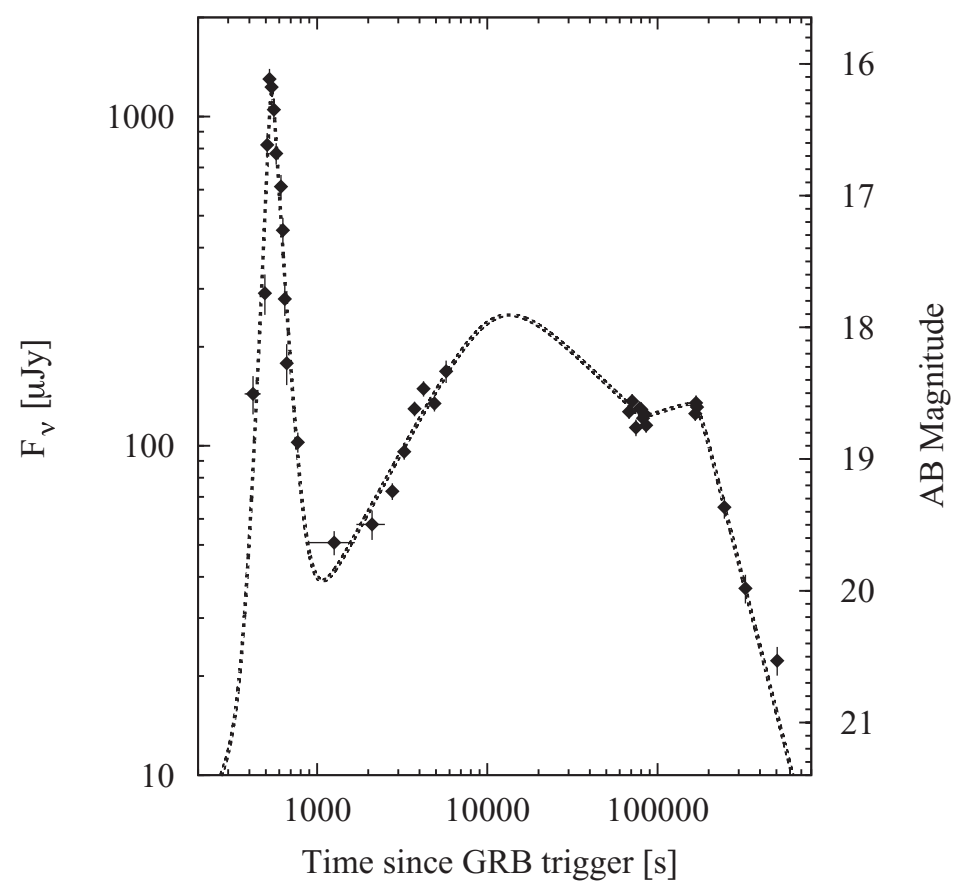

Figure 3. The optical flare of GRB 080129 before its rising afterglow as obtained with the Gamma-ray Optical Near-infrared Detector (GROND). The light-curve is parametrized with a series of smoothly connected power-laws (dashed lines). Super-imposed to the overall afterglow evolution peaking at $10 \mathrm{ks}$, a strong flare with fast rise and fast decay at $t \sim 500 \mathrm{~s}$ is apparent. Adapted from Greiner et al. (2009).

inner engine activity may last or be revived on a timescale of hours or days at least for some bursts.

The early optical afterglow light curve is however not as often and not as strongly affected as the X-rays by flaring episodes. This is readily explained with the spectrum of the flares (peaking in the sub keV to few $\mathrm{keV}$ range), and a bright forward shock component typically dominating the optical emission. Thus, if the emission in the flares is not strong enough with respect to the underlying forward-shock emission, a bright afterglow can easily outshine flare signatures in the optical bands even for very bright X-ray flares.

\section{Jumps - fast rise followed by slow decays}

Remarkably different from the previous light-curve morphologies (fast rising and decaying flares from Section 4, as well as the smooth evolution of the onset of the afterglow from Section 3), is a class of objects characterized by a very fast rise, followed by a sharp turnover to a slow decay. Exemplary of these objects is GRB 081029 (Nardini et al. 2011) or GRB 100621A (Krühler et al. 2011). The excellent coverage in both time and frequency domain for these two events provided by GROND gives a detailed observational picture of their step-like afterglow light-curves.

\subsection{Temporal evolution}

The overall temporal evolution is dominated by the very fast rise (up to $F_{\nu} \propto t^{12}$ ), and can be divided into three phases. The first phase (before $t=3000 \mathrm{~s}$ in Fig. 4 ) shows 


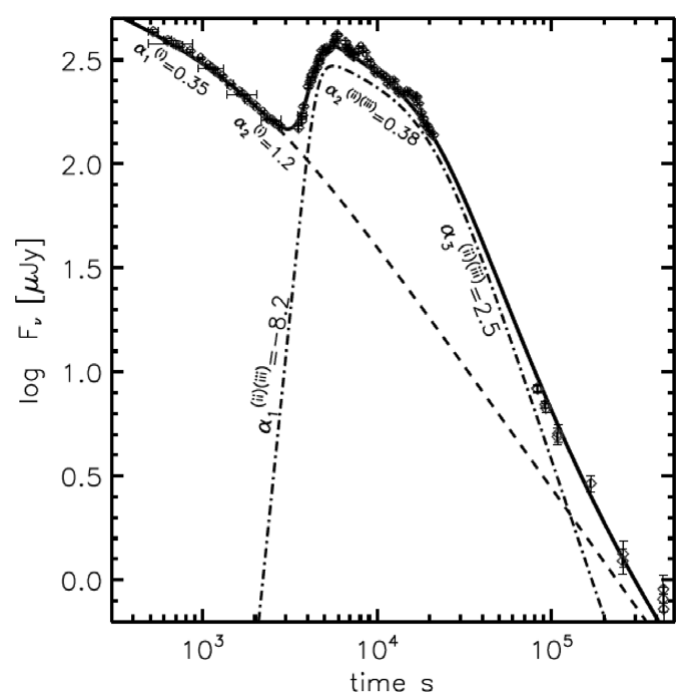

Figure 4. Afterglow light-curve for GRB 081029 as observed with GROND and parametrized using an empirical model consisting of different power-law segments. Taken from Nardini et al. (2011).

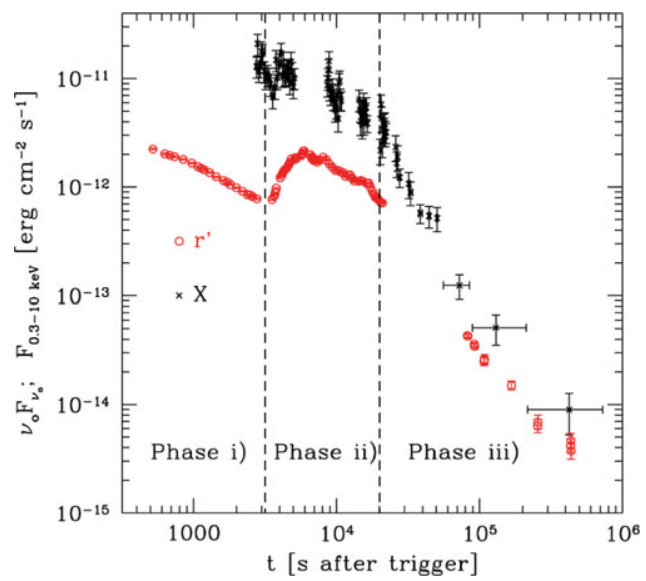

Figure 5. Broad-band behavior of the optical/X-ray afterglow GRB 081029 taken with GROND and the XRT onboard Swift. Taken from Nardini et al. (2011)

a canonical afterglow behavior characterized by a smoothly decaying light-curve with a possible break. Phase 2 displays a sudden rebrightening in all optical/NIR bands, followed by a shallow decay phase. Small scale variations are superimposed onto the shallow decay. The last phase of the afterglow shows a relatively steep $F_{\nu} \propto t^{-2.5}$ decay. An in depth discussion on the specific properties of the afterglow of GRB 081029 can be found in Nardini et al. (2011). This temporal evolution is a common characteristic for the events with a jump feature in their light-curve.

\subsection{Spectral evolution $\&$ broad-band behavior}

Figure 5 shows the optical $r$-band light-curve together with the X-ray data as observed with the XRT. While in the late phases, the optical and X-ray afterglow seem to track each other well, there is a strong discrepancy during the optical jump: there is hardly any 
evolution apparent in the high-energy bands. This is consistent with a strong blue-to-red evolution in the optical/NIR bands during the steep rise. This color evolution observed in the optical/NIR bands is an intrinsic feature of the emission component and is not related to changes of the intervening dust properties. This spectral evolution and broadband behavior seems to be common for all events. In many cases, however, the data are not as detailed as obtained with GROND, and any intrinsic color evolution can not be tested with high accuracy.

\subsection{The class of jumping afterglows}

The literature provides several optical light-curves with similar light curve morphologies, fast rises followed by a shallow decay. They are shown in Figure 6, shifted to rest-frame time, and scaled to $z=1$ for a direct comparison. The shift to $z=1$ includes a rough Kcorrection and intrinsic dust correction. Due to the very different redshifts (from $z=0.5$ to $z=4$ ) the observed $R$-band, where most of the follow-up is performed, is probing very different intrinsic wavelengths.

It is immediately clear, that the jump can appear at very different times, that the rise time is different, and there is only one prominent jump in a single light-curve. The brightness of the underlying afterglow varies over several orders of magnitudes, and the increase in brightness during the jump is typically between one and two magnitudes (corresponding to a factor of 3 to 6 in flux). Selection effects however play a crucial role: the jump component needs to be prominent enough for a burst to enter the sample. The light-curves shown are hence the most extreme examples, and very likely many more cases with less pronounced variability exist.

The apparent dependence on the afterglow is in striking contrast to the true flares as discussed in Section 4, where the flare amplitude is not related to the underlying afterglow. Hence there seems to be a connection between afterglow and the jump components. Furthermore, the steepness of the rise varies significantly, and is not correlated with the time when the jump occurs. The canonical afterglow is typically well established before the rise, and the post-jump decay is shallow and comparable for all the events. In many cases, and if the photometric monitoring is dense and accurate enough, small scale variabilities exist in the shallow decay phase after the steep rise.

\subsection{Jumps at face with theoretical models}

Several theoretical interpretations have been proposed to explain variability in afterglow light-curves (see e.g., Ioka et al. 2005 and references therein). Possible physical explanations for afterglow variability apart from the earlier discussed reverse shocks (Section 2), afterglow onset (Section 3) and flares (Section 4) include inhomogeneities in the circumburst medium (e.g., Wang \& Loeb 2000) or the angular distribution of the energy in the jet (patchy shell model, e.g., Kumar \& Piran 2000) or late energy injection by refreshed shocks (e.g., Rees \& Meszaros 1998) for later flares. Alternatively, it is possible to obtain a chromatic and variable light-curve when decoupling the X-ray and optical afterglow, e.g., through 2-component jets (e.g., Peng et al. 2005) or late prompt models (e.g., Ghisellini et al. 2007, Nardini et al. 2010).

All models, however, have difficulties explaining the very fast rise with a temporal index of up to $F_{\nu} \propto t^{12}$, without invoking a shift of $T_{0}$, i.e., a restarting of the inner engine (Nardini et al. 2011, and references therein). A possible explanation might be present in refreshed shock models, when a late-emitted shell catches up with the decelerating afterglow, and a new forward and reverse shock are formed. These two-shell collisions (Vlasis et al. 2011) could in principle account for the observed features: the steep rise would then be caused by the interaction of the late-and-slow shell with the shocked ISM. 


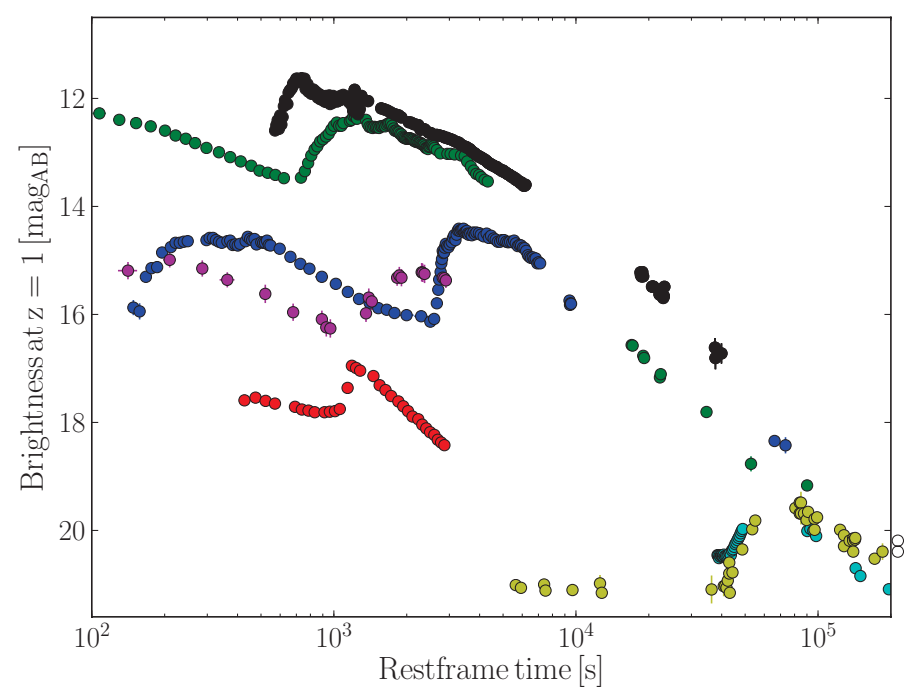

Figure 6. Afterglow light-curves for the sample of jumping afterglows. Data collected from the literature (Pedersen et al. 1998, Stanek et al. 2007, Cenko et al. 2009, Krühler et al. 2011, Nardini et al. 2011) or from the GROND archive.

The morphology of the rise is in this scenario a combination of two emission processes: the increase in energy and Lorentz-factor due to the propagation of the forward shock and the reverse shock traveling back into the late shell. Different steepnesses and amplitudes of the jumps are obtained for different jet geometries, i.e., opening angles, and energy ratios between early and late shells (Vlasis et al. 2011).

\section{Summary}

Despite the large diversity in the morphologies of early optical light curves, they are excellent tools for probing the jet physics. Early optical light curves provide, for example, detailed measurements of the initial Lorentz-factor via the peak of the forward shock. This directly probes the ultra-relativistic nature of the outflow as well as the emission radius of the afterglow. Furthermore, early optical data provide constraints on the role of magnetic fields via the signatures of reverse shocks or lack thereof.

Similar as in many X-ray afterglows, there are also optical/NIR flares superimposed onto the canonical afterglow component. They appear, however, much less frequently in the optical than in the X-ray regime. This is readily explained with the flare spectrum peaking in the few keV range, and the bright afterglow forward shock, dominating the optical emission. These flares indicate, that the inner engine may be active, or revived, up to several hours after the initial burst.

A distinct class of afterglow light-curves is revealed through a very fast rise in brightness followed by a shallow decay phase. Unlike flares, the emission does not drop back immediately to the already established afterglow level. These jumps with rise indices approaching $t^{12}$ present a challenge to canonical models of afterglow variability, and indicate an impulsive release of energy very late after the prompt $\gamma$-ray emission has ceased. This might be caused by refreshed shocks of late or slow shells, catching up with the decelerating forward shock. 
I acknowledge support by the EU under the Marie Curie Programme. I am also very grateful to the GROND team, in particular J. Greiner, M. Nardini and D. A. Kann for constant support and highly valuable input and discussion.

\section{References}

Beloborodov, A. M. 2005, ApJL, 618, L13

Burrows, D. N. et al. 2005, Science, 309, 1833

Gehrels, N. et al. 2004, ApJ, 611, 1005

Cenko, S. B., et al. 2009, ApJ, 693, 1484

Ghisellini, G. et al. 2007, ApJ, 685, 75

Greiner, J. et al. 2008, PASP, 120, 405

Greiner, J. et al. 2009, ApJ, 693, 1912

Ioka, K., et al. 2005, ApJ, 631, 429

Kann, D. A., et al. 2010, ApJ, 720, 1513

Krühler, J. et al. 2008, ApJ, 685, 376

Krühler, J. et al. 2009a, ApJ, 697, 758

Krühler, J. et al. 2009b, A\&A, 508, 593

Krühler, J. et al. 2011, $A \& A$ A, 534, 108

Kumar, P. \& Piran, T. 2000, ApJ, 535, 152

Molinari, E. et al. 2007, A\& A, 469, 13

Nakar, E. \& Piran, T. 2004, MNRAS, 353, 647

Nardini, M. et al. 2010, MNRAS, 403, 1131

Nardini, M. et al. 2011, A\&SA, 535, 57

Panaitescu, A. \& Vestrand, W., T. 2008, MNRAS, 387, 497

Pedersen, H. et al. 1998, ApJ, 496, 311

Peng, F. et al. 2005, ApJ, 626, 966

Perley, D. et al. 2010, MNRAS, 406, 2473

Rees, M. J. \& Meszaros, P. 1998, ApJL, 496, 1

Roming, P. W. A. et al. 2006, ApJ, 652, 1416

Stanek, K. Z. et al. 2007, ApJ, 654, 21

Vlasis, A. et al. 2011, MNRAS, 415, 279

Wang, X. \& Loeb, A. 2000, ApJ, 535, 788

Zhang, B., Kobayashi, S., \& Mészáros, P. 2003, ApJ, 595, 950

\section{Discussion}

Perley: Can you comment on the energetics of the refreshed shock scenario ? It looks to me that in order to increase the brightness by a factor of 3 or more, the radiated energy integrated over time must increase by a similar factor.

KRÜHLER: In the shown examples, arguably the most extreme ones, the energy carried by the second shell is few times larger than in the initial shell.

IoKA: What is the time-seperation for the two shells ? The usual refreshed shock model with simultaneous ejection would be impossible to reproduce in the early fast rise.

KRÜHLER: The time separation is of order several thousand seconds, so also the refreshedshock scenario needs a late time activity of the central engine. The fast rise is indeed challenging to explain in canonical models of afterglow variability without invoking a late engine activity. 